\title{
Single-Molecule Long-Read Transcriptome Dataset of Halophyte Halogeton glomeratus
}

\author{
Juncheng Wang ${ }^{1,2+}$, Lirong Yao ${ }^{1,2+}$, Baochun $\mathrm{Li}^{1,3}$, Yaxiong Meng ${ }^{1,2}$, Xiaole $\mathrm{Ma}^{1,2}$ and \\ Huajun Wang ${ }^{1,2 *}$
}

${ }^{1}$ Gansu Provincial Key Lab of Aridland Crop Science, Gansu Key Lab of Crop Improvement and Germplasm Enhancement, Gansu Agricultural University, Lanzhou, China, ${ }^{2}$ Department of Crop Genetics and Breeding, College of Agronomy, Gansu Agricultural University, Lanzhou, China, ${ }^{3}$ Department of Botany, College of Life Sciences and Technology, Gansu Agricultural University, Lanzhou, China

Keywords: Halophyte, H. glomeratus, Iso-Seq, transcriptome, salt stress

\section{INTRODUCTION}

Soil salinization has become a major challenge for sustainable development of global agriculture. As a result, cultivation of salt-tolerant crop varieties has become a focus of plant breeding. However,

OPEN ACCESS

Edited by:

Graziano Pesole,

Università degli studi di Bari Aldo

Moro, Italy

Reviewed by:

RaffaEle A. Calogero,

Università degli Studi di Torino, Italy

Matteo Chiara,

Università degli Studi di Milano, Italy

${ }^{*}$ Correspondence:

Huajun Wang

whuajun@yahoo.com

${ }^{\dagger}$ These authors have contributed equally to this work.

Specialty section: This article was submitted to

RNA,

a section of the journal

Frontiers in Genetics

Received: 25 August 2017 Accepted: 20 November 2017 Published: 01 December 2017

Citation:

Wang J, Yao L, Li B, Meng Y, MaX and Wang $H$ (2017) Single-Molecule Long-Read Transcriptome Dataset of

Halophyte Halogeton glomeratus.

Front. Genet. 8:197.

doi: 10.3389/fgene.2017.00197 development of effective breeding strategies would be significantly enhanced by improving our understanding of salt tolerance mechanisms in plants and identifying genes required for adaptation (Flowers and Colmer, 2008; Shabala, 2013; Ismail and Horie, 2017).

$\mathrm{Na}^{+}$is not essential for plant survival; in fact, there are many halophytes whose growth is improved upon the addition of a certain degree of $\mathrm{NaCl}$ (Bose et al., 2014). As a result of this unique survival strategy, halophytes have been used to elucidate a series of mechanisms that use to cope with salt stress. In particular, succulent halophytes, a representative type of anatomical adaptation in plants by increasing cell size due to the expansion of vacuole volume under salinity, have been suggested to take advantage of tissue- and organelle-specific ion compartmentalization to maintain high $\mathrm{K}^{+} / \mathrm{Na}^{+}$ratios in response to high salinity (Munns and Tester, 2008; Shabala and Mackay, 2011). Furthering our molecular understanding of the ion transport systems and compartmentalization mechanisms used by halophytes is crucial for mapping the complex salt tolerance network in plants in order to optimize breeding of salt-tolerant crops.

Halogeton glomeratus (H. glomeratus), an annual, succulent halophyte, is a typical salt-tolerant halophyte with highly succulent leaves that has been used to characterize the physiological, cellular, and molecular mechanisms involved in the response to salt stress (Wang et al., 2015a,b, 2016).

The majority of next-generation transcriptome sequencing (NGS) technologies, or RNA sequencing (RNA-Seq) are not capable of assembling full-length transcripts due to the short lengths of sequencing-reads, which are likely to produce partial reference transcriptomes (Martin and Wang, 2011). For the RNA-Seq of non-model organisms, one of the principal strategies is de novo assembly. In this way, we could retrieve plenty of transcripts by piecing together short, low-quality sequencing-reads in a more economical approach, and provide a new opportunity to cognize the transcriptome characteristics of non-model organisms, but the disadvantages of the de novo assembly are obvious, such as a greater sequencing depth, highly sensitive to sequencing errors, presence of chimeric molecules in the sequencing dataset, and difficult to distinguish highly similar transcripts (Martin et al., 2010; Martin and Wang, 2011). In a word, it is still challenging to construct of a comprehensive transcriptome from short sequencing-reads. However, recent development of Pacific Biosciences (PacBio) long-read technologies for transcriptome sequencing (Iso-Seq) can overcome those limitations to capture the full catalog of transcripts and their variants (Sharon et al., 2013; Minoche et al., 2015). Thus, for the first time, we applied the Iso-Seq 
protocol to analyze the $H$. glomeratus transcriptome. These findings serve to improve $H$. glomeratus transcriptome annotation and provide abundant gene resources related to improve salt tolerance in plants.

\section{VALUE OF THE DATA}

1. Halogeton glomeratus is not only highly valuable for the characterization of salt tolerance mechanisms in halophytes, but may also serve as a source of stress tolerance genes for plant breeding.

2. So far, there is lack of well-characterized transcriptomic profiling of $H$. glomeratus. Here, we provided the first comprehensive transcriptome analysis using single-molecule sequencing.

3. These data will help elucidate salt tolerance mechanisms of H. glomeratus and provide salt-tolerant gene resources for developing stress-tolerant plants.

\section{DATA}

\section{Transcriptome Plant Materials and $\mathrm{NaCl}$ Treatments}

Halogeton glomeratus seedlings were grown in plastic pots in a growth chamber and were irrigated daily with half-strength Hoagland's nutrient solution as previously described by Wang et al. (2015a). One month after germination (August 10, 2016), two independent experiments were conducted to assess different durations of salt stress. For the first experiment, seedlings were transferred to half-strength Hoagland's nutrient solution supplemented with $0,100,200$, or $400 \mathrm{mM} \mathrm{NaCl}$ for a period of 3 days. The leaf samples were collected for each condition. For the second experiment, the seedlings were transferred to half-strength Hoagland's nutrient solution supplemented with $200 \mathrm{mM} \mathrm{NaCl}$ for $0,2,6,24$, or $72 \mathrm{~h}$. The root samples were collected for each condition and were rinsed with distilled water. All samples were frozen in liquid $\mathrm{N}_{2}$ immediately.

\section{Generation of a Barcoding Library and Single-Molecule Sequencing}

After grinding the tissue samples, total RNA was extracted from each sample using the RNeasy Plant Mini kit (Qiagen; Hilden, Germany) and quantified using an Agilent 2100 bioanalyzer. The total RNA from the 9 samples were pooled for sequencing of an $H$. glomeratus reference transcriptome using single-molecule long-read technology (Iso-Seq) via the PacBio RS II sequencing platform.

In brief, total RNA (10 ng) was reverse transcribed using the SMARTer PCR cDNA Synthesis Kit (Clontech; Mountain View, CA, USA). After polymerase chain reaction (PCR) optimization, large-scale PCR reactions were performed to synthesize secondstrand cDNA, which was subjected to BluePippin ${ }^{\mathrm{TM}}$ size selection using the following bins: 1-2, 2-3, 3-6, and 5-10 kb. After size selection, another round of amplification was performed using 12 PCR cycles. The amplified and size-selected cDNA products were used to generate SMRTbell template libraries for sequencing based on the Iso-Seq protocol. A total of 12 SMRT cells (Table 1) were sequenced on a PacBio RS II platform (Completed on January 2, 2017). Raw sequence reads (FASTQ format) are available through the NCBI database (https://www.ncbi.nlm. nih.gov/) with the following accession information (Data was released on September 22, 2017, size is $1.6 \mathrm{~Gb}$ ):

BioProject ID: PRJNA359784.

BioSample ID: SAMN06298282.

\section{Bioinformatics Analysis of Isoform Sequencing}

Raw SMRT sequencing reads were classified as full-length (FL) non-chimeric, FL chimeric, non-FL, or short reads based on detection of $5^{\prime}$ - and $3^{\prime}$-primer and polyA-tail sequences using SMRT Analysis v2.3.0 (http://smrt-analysis.readthedocs.io/en/ latest/SMRT-Analysis-Software-Installation-v2.3.0/). Only reads with both $5^{\prime}$ - and $3^{\prime}$-primers and polyA-tail sequences were considered to be FL. FL non-chimeric reads were clustered into consensus sequence (CS) reads using the ICE (Interative Clustering and Error Correction) algorithm and further polished using Quiver. Finally, high quality CS reads from each library were merged, and redundancy was removed to identify unique isoforms present in the $H$. glomeratus transcriptome using the Cd-hit program (Li and Godzik, 2006) for further analysis. All of the isoform sequences are publicly available on Figshare at https://figshare.com/articles/Single-molecule_long-read_ transcriptome_dataset_of_halophyte_Halogeton_glomeratus/ 5345464 .

A total of 433,420 reads of insert (ROIs) were generated. Fulllength (FL) non-chimeric transcripts made up $57.96 \%$ of the $1-2 \mathrm{~kb}$ transcripts, $50.05 \%$ of the $2-3 \mathrm{~kb}$ transcripts, $58.28 \%$ of the $3-6 \mathrm{~kb}$ transcripts, and $35.33 \%$ of the $5-10 \mathrm{~kb}$ transcripts, and the libraries were characterized by average lengths of $1,657,2,848,3,835$, and 6,221 bp, respectively (Table 1). The FL non-chimeric transcripts were processed using Quiver to yield 64,910 high quality consensus isoforms. After additional merging and discarding of redundancies, we obtained 54,835 consensus isoforms with a mean length of 2,663 nt and a N50 length of 2,937 nt (Figures 1A,B).

The isoform sequences were used as queries for sequence homology searches $(E<0.0001)$ performed with Blast, Blast2GO (Conesa et al., 2005), and InterProScan5 (Quevillon et al., 2005) to identify functional annotation terms from the nonredundant protein (NR), non-redundant nucleotide (NT), Gene Ontology (GO), Clusters of Orthologous Groups (COG), Kyoto Encyclopaedia of Genes and Genomes (KEGG), SwissProt, and Interpro databases. For isoform coding DNA sequence (CDS) predictions, we selected the segment of the isoform that best mapped to the functional databases as its CDS. For isoforms that were not annotated in the databases, we used ESTScan (Iseli et al., 1999) to predict CDSs. The predict CDSs of isoforms are publicly available on Figshare at: https://figshare.com/ articles/Single-molecule_long-read_transcriptome_dataset_of_ halophyte_Halogeton_glomeratus/5345563.

A total of 53,230 (97.07\%) isoforms were matched to known genes, and 52,141 (95.07\%) isoforms were identified to have 
TABLE 1 | Proportion of SMRT reads containing cDNA primers and poly(A) tail and their classification.

\begin{tabular}{|c|c|c|c|c|c|c|c|}
\hline Database & $\begin{array}{c}\text { Cell } \\
\text { number }\end{array}$ & All reads & $\begin{array}{l}\text { Five prime reads } \\
\qquad(\%)\end{array}$ & $\begin{array}{l}\text { Three prime reads } \\
(\%)\end{array}$ & $\begin{array}{l}\text { Poly-A reads } \\
\text { (\%) }\end{array}$ & $\begin{array}{l}\text { Full-length non-chimeric } \\
\text { reads }(\%)\end{array}$ & $\begin{array}{c}\text { Full-length non-chimeric read } \\
\text { length (bp) }\end{array}$ \\
\hline $1-2 \mathrm{~kb}$ & 4 & 152,748 & 68.01 & 69.89 & 69.12 & 57.96 & 1,657 \\
\hline $2-3 \mathrm{~kb}$ & 4 & 176,650 & 60.10 & 62.56 & 62.02 & 50.05 & 2,848 \\
\hline $3-6 \mathrm{~kb}$ & 2 & 52,148 & 72.13 & 74.09 & 73.44 & 58.28 & 3,835 \\
\hline $5-10 \mathrm{~kb}$ & 2 & 51,874 & 53.04 & 61.68 & 59.97 & 35.33 & 6,221 \\
\hline
\end{tabular}

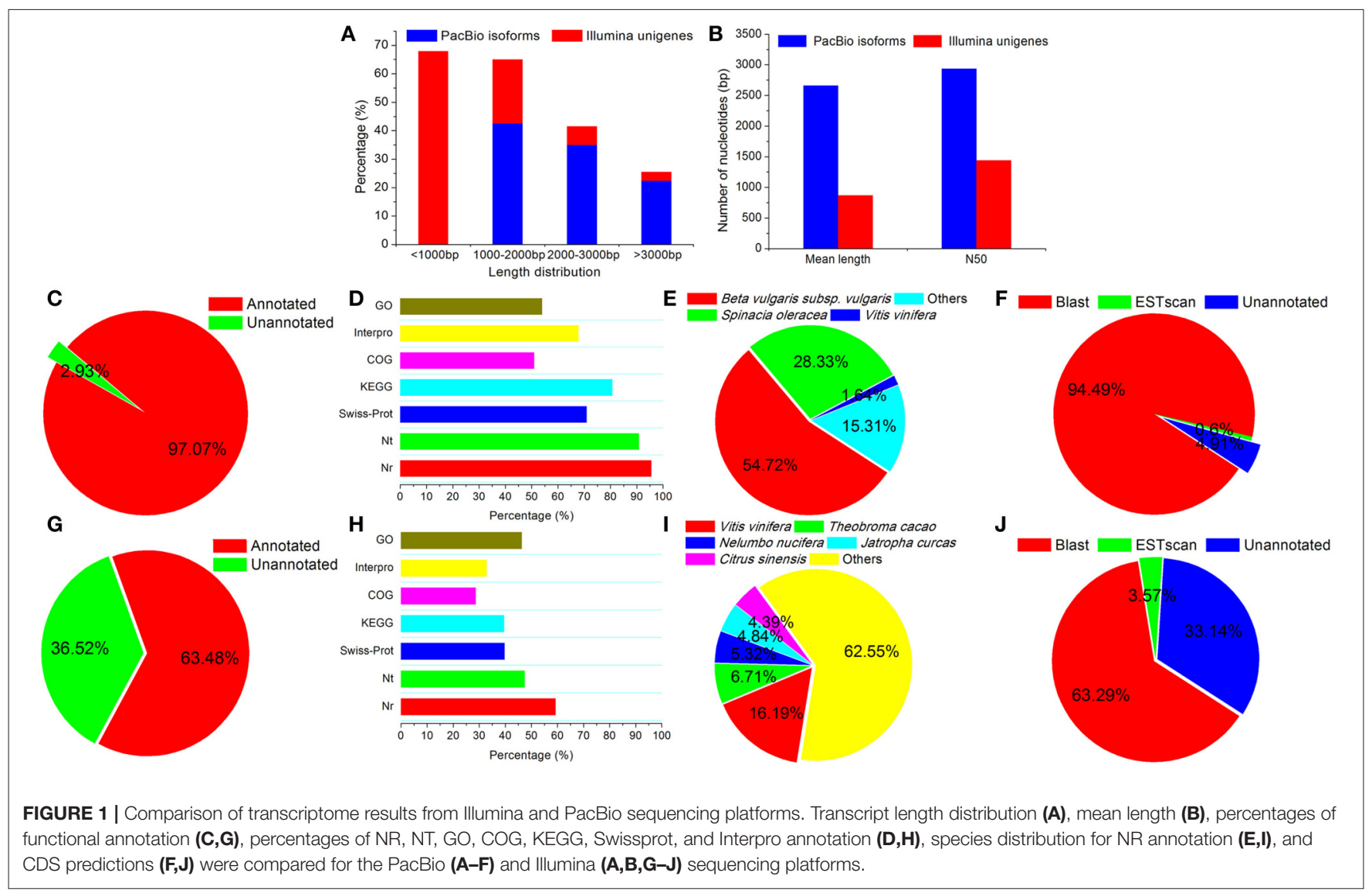

intact coding DNA sequences (CDS) (Figures 1C-F). At the same time, our previous Illumina RNA-Seq data of $H$. glomeratus (Wang et al., 2015b) was re-annotated by the update versions of the database used in the present Iso-Seq transcriptome sequencing, including NR, NT, GO, COG, KEGG, SwissProt, and Interpro databases. In comparison to the results from Illumina RNA-Seq analysis of $H$. glomeratus, the length distribution, functional annotation, and coding sequence quantity of the Iso-Seq transcripts were significantly improved (Figure 1). In particular, with respect to the species distribution of annotation from the NR database, we found that $98.31 \%$ of the annotated isoforms showed the highest similarity to sequences from the three most prevalent species: beta vulgaris subsp. vulgaris (54.72\%), spinacia oleracea (28.33\%), and vitis vinifera $(15.31 \%)$ (Figure 1E). Like H. glomeratus, beta vulgaris subsp. vulgaris and spinacia oleracea belongs to Chenopodiaceae. In contrast, for unigenes identified using Illumina RNA-Seq, the top species identified was vitis vinifera (16.19\%), and none of the top five species belong to Chenopodiaceae (Figure 1I). Besides, to better understand the advantages of the PacBio Iso-Seq reference transcriptome, we mapped the Illumina de novo assembled transcripts $(50,267)$ to the Iso-Seq consensus transcripts $(54,835)$. Among Iso-Seq consensus transcripts, a total of 21,008 exhibited homology to Illumina de novo assembled 36,548 transcripts $(E<0.0001)$, of which 8,236 (39.20\%) exhibited homology with at least two Illumina de novo assembled transcripts, including 4,668 (22.22\%), 1,860 (8.85\%) and 1,708 (20.74\%) exhibited homology with two, three, and the more Illumina de novo assembled transcripts, respectively (Supplementary Figure 1). These results are successfully consistent with comprehensive assembly and annotation of a reference $H$. glomeratus transcripts using Iso-Seq technology that is substantially more contigous and contains a higher proportion of intact CDS. 


\section{AUTHOR CONTRIBUTIONS}

HW designed the experiments. JW, LY, BL, YM, and XM collected the samples and performed the experiments. JW, LY, and BL analyzed RNA-Seq data. JW and LY wrote the manuscript. All authors read and approved the final manuscript.

\section{ACKNOWLEDGMENTS}

This work was supported by National Basic Research Program of China (973 program, 2014CB160313), China Agriculture Research System (CARS-05), National Natural Science

\section{REFERENCES}

Bose, J., Rodrigo-Moreno, A., and Shabala, S. (2014). ROS homeostasis in halophytes in the context of salinity stress tolerance. J. Exp. Bot. 65, 2808-2837. doi: $10.1093 /$ jxb/ert430

Conesa, A., Götz, S., García-Gómez, J. M., Terol, J., Talón, M., and Robles, M. (2005). Blast2GO: a universal tool for annotation, visualization and analysis in functional genomics research. Bioinformatics 21, 3674-3676. doi: 10.1093/bioinformatics/bti610

Flowers, T. J., and Colmer, T. D. (2008). Salinity tolerance in halophytes. New Phytol. 179, 945-963. doi: 10.1111/j.1469-8137.2008.02531.x

Iseli, C., Jongeneel, C. V., and Bucher, P. (1999). ESTscan: a program for detecting, evaluating, and reconstructing potential coding regions in EST sequences. Proc. Int. Conf. Intell. Syst. Mol. Biol. 99, 138-148.

Ismail, A. M., and Horie, T. (2017). Genomics, physiology, and molecular breeding approaches for improving salt tolerance. Annu. Rev. Plant Biol. 68, 405-434. doi: 10.1146/annurev-arplant-042916-040936

Li, W., and Godzik, A. (2006). Cd-hit: a fast program for clustering and comparing large sets of protein or nucleotide sequences. Bioinformatics 22, 1658-1659. doi: 10.1093/bioinformatics/btl158

Martin, J. A., and Wang, Z. (2011). Next-generation transcriptome assembly. Nat. Rev. Genet. 12, 671-682. doi: 10.1038/nrg3068

Martin, J., Bruno, V. M., Fang, Z., Meng, X., Blow, M., Zhang, T., et al. (2010). Rnnotator: an automated de novo transcriptome assembly pipeline from stranded RNA-Seq reads. BMC Genomics 11:663. doi: 10.1186/1471-2164-11-663

Minoche, A. E., Dohm, J. C., Schneider, J., Holtgräwe, D., Viehöver, P., Montfort, M., et al. (2015). Exploiting single-molecule transcript sequencing for eukaryotic gene prediction. Genome Biol. 16:184. doi: 10.1186/s13059-015-0729-7

Munns, R., and Tester, M. (2008). Mechanisms of salinity tolerance. Plant Biol. 59, 651-681. doi: 10.1146/annurev.arplant.59.032607.092911
Foundation of China (No. 31460347), and Fostering Foundation for the Excellent Ph.D. Dissertation of Gansu Agricultural University (YBPY2014001).

\section{SUPPLEMENTARY MATERIAL}

The Supplementary Material for this article can be found online at: https://www.frontiersin.org/articles/10.3389/fgene. 2017.00197/full\#supplementary-material

Supplementary Figure 1 | Statistics of the correspondence between the Illumina unigenes and PacBio reference transcripts of $H$. glomeratus transcriptome.

Quevillon, E., Silventoinen, V., Pillai, S., Harte, N., Mulder, N., Apweiler, R., et al. (2005). InterProScan: protein domains identifier. Nucleic Acids Res. 33, 116-120. doi: 10.1093/nar/gki442

Shabala, S. (2013). Learning from halophytes: physiological basis and strategies to improve abiotic stress tolerance in crops. Ann. Bot. 112, 65-69. doi: $10.1093 / \mathrm{aob} / \mathrm{mct} 205$

Shabala, S., and Mackay, A. (2011). Ion transport in halophytes. Adv. Bot. Res. 57, 151-187. doi: 10.1016/B978-0-12-387692-8.00005-9

Sharon, D., Tilgner, H., Grubert, F., and Snyder, M. (2013). A single-molecule long-read survey of the human transcriptome. Nat. Biotechnol. 31, 1009-1014. doi: 10.1038/nbt.2705

Wang, J., Meng, Y., Li, B., Ma, X., Lai, Y., Si, E., et al. (2015a). Physiological and proteomic analyses of salt stress response in the halophyte Halogeton glomeratus. Plant Cell Environ. 38: 655-669. doi: 10.1111/pce.12428

Wang, J., Li, B., Meng, Y., Ma, X., Lai, Y., Si, E., et al. (2015b). Transcriptomic profiling of the salt-stress response in the halophyte Halogeton glomeratus. BMC Genomics 16:169. doi: 10.1186/s12864-015-1373-Z

Wang, J., Yao, L., Li, B., Meng, Y., Ma, X., Lai, Y., et al. (2016). Comparative proteomic analysis of cultured suspension cells of the halophyte Halogeton glomeratus by iTRAQ provides insights into response mechanisms to salt stress. Front. Plant Sci. 7:110. doi: 10.3389/fpls.2016.00110

Conflict of Interest Statement: The authors declare that the research was conducted in the absence of any commercial or financial relationships that could be construed as a potential conflict of interest.

Copyright (C) 2017 Wang, Yao, Li, Meng, Ma and Wang. This is an open-access article distributed under the terms of the Creative Commons Attribution License (CC BY). The use, distribution or reproduction in other forums is permitted, provided the original author(s) or licensor are credited and that the original publication in this journal is cited, in accordance with accepted academic practice. No use, distribution or reproduction is permitted which does not comply with these terms. 